\title{
Translation initiation by the c-myc mRNA internal ribosome entry sequence and the poly(A) tail
}

\author{
CHRISTIAN THOMA, ${ }^{1}$ SVEN FRATERMAN, MARC GENTZEL, MATTHIAS WILM, and MATTHIAS W. HENTZE \\ European Molecular Biology Laboratory (EMBL), 69117 Heidelberg, Germany
}

\begin{abstract}
Eukaryotic mRNAs possess a poly(A) tail that enhances translation via the ${ }^{7} \mathrm{mGpppN}$ cap structure or internal ribosome entry sequences (IRESs). Here we address the question of how cellular IRESs recruit the ribosome and how recruitment is augmented by the poly(A) tail. We show that the poly(A) tail enhances $48 \mathrm{~S}$ complex assembly by the c-myc IRES. Remarkably, this process is independent of the poly(A) binding protein (PABP). Purification of native $48 \mathrm{~S}$ initiation complexes assembled on c-myc IRES mRNAs and quantitative label-free analysis by liquid chromatography and mass spectrometry directly identify eIFs 2, 3, 4A, 4B, $4 \mathrm{GI}$, and 5 as components of the c-myc IRES $48 \mathrm{~S}$ initiation complex. Our results demonstrate for the first time that the poly(A) tail augments the initiation step of cellular IRES-driven translation and implicate a distinct subset of translation initiation factors in this process. The mechanistic distinctions from cap-dependent translation may allow specific translational control of the C-myc mRNA and possibly other cellular mRNAs that initiate translation via IRESs.
\end{abstract}

Keywords: translation initiation; cellular IRES; poly(A) tail; quantitative mass spectrometry; native RNPS

\section{INTRODUCTION}

Most eukaryotic mRNAs initiate translation via the $5^{\prime}$ $\mathrm{m}^{7} \mathrm{GpppN}$ cap structure and the 3' poly(A) tail (Gallie 1991; Tarun and Sachs 1995; Preiss and Hentze 1998; Gingras et al. 1999). A bridging complex between the capbinding protein eIF4E, the adaptor protein eIF4G (I or II), and PABP bound to the $3^{\prime}$ poly(A) tail can mediate circularization of the mRNA (Wells et al. 1998). Biochemically, multiple mechanisms may contribute to enhance translation by: (1) promoting small ribosomal subunit recruitment via the $5^{\prime}$-cap structure (Tarun and Sachs 1995; Kahvejian et al. 2005); (2) stimulating 60S ribosomal subunit joining (Sachs and Davis 1989; Munroe and Jacobson 1990; Searfoss et al. 2001); and/or (3) facilitating translation termination and ribosome recycling (Uchida et al. 2002).

Translation can also initiate in a $5^{\prime}$-end-independent way mediated by internal ribosomal entry sequences (IRESs). IRESs are cis-acting RNA sequences first discovered in picornaviral RNAs (Jang et al. 1988; Pelletier and Sonenberg

\footnotetext{
${ }^{1}$ Present address: Department of Medicine II, University Hospital of Freiburg, Hugstetterstrasse 55, 79106 Freiburg, Germany.

Reprint requests to: Matthias W. Hentze, European Molecular Biology Laboratory (EMBL), Meyerhofstrasse 1, 69117 Heidelberg, Germany; hentze@embl.de; fax: 49-6221-387518.

Article published online ahead of print. Article and publication date are at http://www.rnajournal.org/cgi/doi/10.1261/rna.1043908.
}

1988) and also found in the $5^{\prime}$-untranslated region (UTR) of some cellular mRNAs (Sarnow 1989; Hellen and Sarnow 2001; Gilbert et al. 2007). Viral IRESs mediate translation by multiple mechanisms that differ in their requirements for translation initiation factors. In encephalomyocarditis virus (EMCV) IRES-mediated translation, an interaction of eIF4G and eIF4A with the IRES, in concert with the canonical eIFs 2,3 , and $4 \mathrm{~B}$, is necessary to recruit $40 \mathrm{~S}$ ribosomes to the IRES. The hepatitis $\mathrm{C}$ virus (HCV) IRES depends on the ternary eIF2/GTP/initiator tRNA complex and on eIF3 for $48 \mathrm{~S}$ complex formation, but does not require any eIF4 family members (Hellen and Sarnow 2001). Most remarkably, the cricket paralysis virus (CrPV) IRES can assemble $80 \mathrm{~S}$ ribosomes without any eIFs (Wilson et al. 2000). In addition to the distinctive requirements for canonical initiation factors, noncanonical initiation factors, known as IRES trans-acting factors (ITAFs), have been implicated in enhancing initiation of several viral IRESs (Dorner et al. 1984; Jackson and Kaminski 1995; Kolupaeva et al. 2007). The $\operatorname{poly}(\mathrm{A})$ tail also stimulates translation via some viral IRESs (polio-, EMCV-, coxackievirus [CV] and hepatitis A virus [HAV]) (Bergamini et al. 2000; Khaleghpour et al. 2001; Michel et al. 2001; Svitkin et al. 2001; Bradrick et al. 2007). Similar to cap-dependent translation, the stimulatory effect of the poly(A) tail on viral IRES-driven translation appears to involve the eIF4G-PABP interaction (Michel et al. 2001; Svitkin et al. 2001). Numerous cellular IRESs allow protein synthesis under conditions when cap-dependent 
translation is compromised, for example during apoptosis, mitosis, and different forms of stress (Cornelis et al. 2000; Pyronnet et al. 2000; Stoneley et al. 2000; Fernandez et al. 2001; Subkhankulova et al. 2001; Kim et al. 2003; Braunstein et al. 2007; Gilbert et al. 2007) and some 3\% of the cellular transcriptome continues to be translated when cap-dependent translation is inhibited by poliovirus infection (Johannes and Sarnow 1998). In several cases cellular IRES-driven translation has been attributed to the activity of ITAFs (Pilipenko et al. 2000; Stoneley and Willis 2004; Cobbold et al. 2008). Their molecular mode of action remains to be clearly defined.

The transcript encoding the $\mathrm{c}-\mathrm{Myc}$ protein belongs to the mRNAs that are actively translated during apoptosis, mitosis, or cellular stress when cap-dependent translation is compromised (Stoneley et al. 2000; Subkhankulova et al. 2001; Kim et al. 2003). The expression of c-myc is critical for cell proliferation/differentiation and apoptosis (Evan and Littlewood 1998; Eisenman 2001), and the c-myc IRES has been implicated in cancer: Increased c-myc expression in cells derived from patients suffering from multiple myeloma has been attributed to somatic mutations within the IRES (Chappell et al. 2000).

In contrast to viral IRESs, very little is known about the mechanism of cellular IRES function. In particular, it is presently not understood how cellular IRESs recruit the ribosome. Using a cell-free translation system derived from HeLa cells and an in vivo assay based on RNA transfections, we showed that the poly(A) tail enhances IRES-directed translation of the c-myc and BiP mRNAs in vitro and in vivo (Thoma et al. 2004a). Here, we address the mechanistic basis for this enhancement. We show that the poly(A) tail stimulates $48 \mathrm{~S}$ initiation complex assembly via the c-myc IRES. Remarkably, the PABP/eIF4G interaction is dispensable for this enhancement, and the enhancer function of the poly (A) tail is therefore distinct from its role in the activation of cap-dependent translation initiation. Direct purification and quantitative analysis of native cmyc IRES 48S initiation complexes identifies a distinct subset of translation initiation factors that includes eIF4GI but lacks eIF4GII.

\section{RESULTS}

\section{PABP and intact elF4G are dispensable for the poly(A) tail-mediated enhancement of c-myc IRES-driven translation}

To examine the mechanism of translation initiation by cellular IRESs, we developed a HeLa cell-derived in vitro translation system that supports cellular IRES function (Thoma et al. 2004a,b). We found that the poly(A) tail enhances cellular (c-myc and BiP) IRES-mediated translation and showed that-in contrast to cap-dependent translation-the stimulatory effect of the poly(A) tail on cellular IRESs requires neither PABP nor intact eIF4G (Fig. 1; Thoma et al. 2004a). PABP has been proposed to stimulate cap-dependent translation initiation and ribosomal recycling (Uchida et al. 2002; Kahvejian et al. 2005). There is no information regarding the step of cellular IRESdriven translation that is affected by the poly(A) tail.

Since the c-myc IRES is active in apoptotic cells and apoptosis causes cleavage of both eIF4G I and II, we decided to study the c-myc IRES and the enhancer function of the poly(A) tail in HeLa extracts where the eIF4Gs were proteolytically cleaved (Marissen and Lloyd 1998; Bushell et al. 1999; Stoneley et al. 2000). In addition, we wanted to directly evaluate the role of PABP, as earlier studies conducted in the presence of intact eIF4G indicated that PABP was dispensable for c-myc IRES enhancement by the poly(A) tail (Thoma et al. 2004a).

PABP was depleted from the translation extracts by PAIP2-mediated affinity chromatography (Svitkin and Sonenberg 2004) by more than $90 \%$. (Fig. 1A, right panel). To functionally confirm the effectiveness of both proteolytic eIF4G cleavage and PABP depletion, we evaluated the translation of ${ }^{7} \mathrm{mGpppG}$-capped mRNAs with or without an poly(A) tail. Figure 1C shows that eIF4G cleavage (Fig. 1C, lanes 5,6), PABP-depletion (Fig. 1C, lanes 3,4 ), and the combination of the two (Fig. 1C, lanes 7,8 ) strongly inhibit the stimulation of cap-dependent translation by the poly(A) tail (Fig. 1C, cf. lanes 1 and 2). We then evaluated the function of PABP in the poly(A) enhancement of c-myc IRES-driven translation when eIF4G is cleaved. In sharp contrast to cap-dependent translation, c-myc IRES-driven translation is fully enhanced by the poly(A) tail, even following eIF4G cleavage and PABP-depletion (Fig. 1B, cf. lanes 7,8 and 5,6). PABP depletion even stimulates c-myc IRES activity in these extracts (Fig. 1B, cf. lanes 6 and 8). These results show that the poly(A) tail enhances c-myc IRES translation independently of PABP and intact eIF4G.

\section{The poly(A) tail enhances translation initiation}

Next, we aimed to identify the mechanism underlying this enhancer function of the poly(A) tail. Initiation is frequently rate limiting and targeted by multiple control mechanisms. To investigate whether the initiation step of c-myc IRES translation is augmented by the poly(A) tail, we analyzed ribosome assembly on c-myc mRNAs in sucrose density gradient experiments. To optimize resolution, we created a shortened form of the c-myc IRES reporter mRNA by replacing the luciferase open reading frame (ORF) with a synthetic short ORF (Fig. 2A; Gebauer et al. 2003). We validated that this shortened c-myc reporter mRNA is also translated via its IRES (Fig. 2B) and that translation of the c-myc IRES/short ORF transcripts is enhanced by the presence of a poly $(\mathrm{A})$ tail (Fig. 2B). 
A

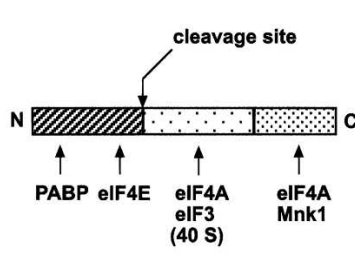

elF4G I

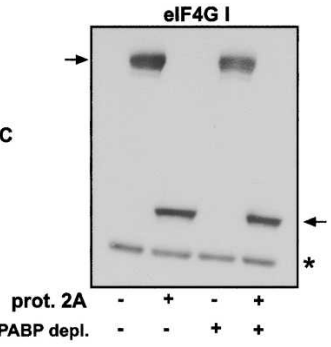

depleted: non mock PABP

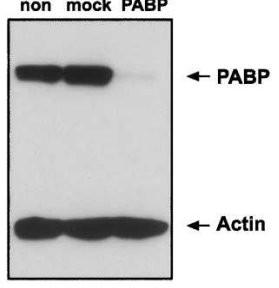

B
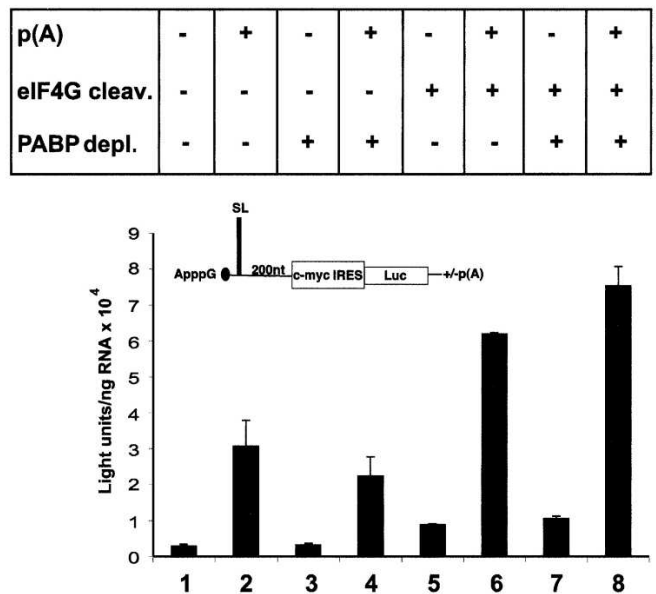

C

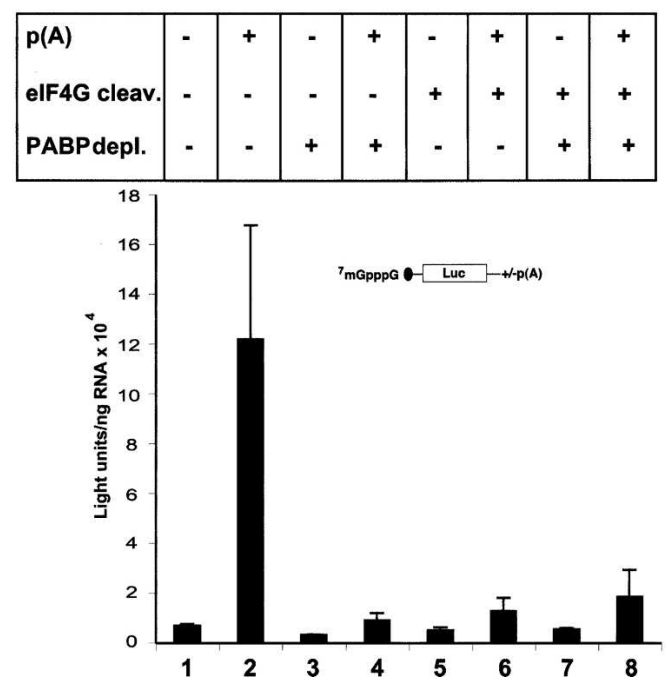

FIGURE 1. Enhancement of c-myc IRES-mediated translation is independent of intact eIF4G and PABP. (A) Domain structure of eIF4G with protein binding sites and the protease $2 \mathrm{~A}$ cleavage site indicated by arrows (left panel). Western blot analysis of eIF4GI in the indicated extracts (middle panel) and PABP in non-, mock, or PABP-depleted HeLa extracts (right panel). The positions of intact proteins and the respective cleavage products are indicated by arrows. Asterisk denotes cross-reactive nonspecific bands. $(B, C)$ The indicated mRNAs were translated in mock-, PABP-depleted, protease 2A-treated, or PABP-depleted and protease 2Atreated HeLa cell extracts. The data represent the average of three experiments with error bars indicating the standard deviation from the mean.

Translation initiation reactions were performed in the presence of the translation elongation inhibitor cycloheximide, which limits translation initiation exclusively to single rounds, thus excluding potential secondary effects

caused by ribosome recycling. As expected, ribosomal $80 \mathrm{~S}$ complexes assemble on c-myc IRES mRNAs (Fig. $2 \mathrm{C}$, fractions $3-5$, blue line). By contrast, $80 \mathrm{~S}$ peak formation is almost absent on c-myc IRES mRNAs lacking the poly(A) tail, demonstrating directly that the $\operatorname{poly}(\mathrm{A})$ tail stimulates $80 \mathrm{~S}$ ribosomal complex formation and hence translation initiation on c-myc IRES mRNAs (Fig. 2C, fractions 3-5, cf. pink and blue lines). Changes in mRNA stability are not responsible for the observed stimulation of c-myc IRES function by the poly(A) tail (Fig. 2D). Recall that these experiments were performed in extracts following eIF4G cleavage, implying that the observed poly(A) tail enhancement of cellular IRES-mediated initiation is independent of intact eIF4G.

The poly(A) tail stimulates $48 \mathrm{~S}$ initiation complex formation via the c-myc IRES

Multiple mechanisms contribute to how poly(A) tails stimulate cap-dependent translation, including both $48 \mathrm{~S}$ initiation complex formation and 60S ribosomal subunit joining (Sachs and Davis 1989; Munroe and Jacobson 1990; Tarun and Sachs 1995; Searfoss et al. 2001; Kahvejian et al. 2005). To determine how the poly(A) tail stimulates c-myc IRES-directed translation initiation, we analyzed its effect on $48 \mathrm{~S}$ complex formation. Because GTP hydrolysis is essential for 605 ribosomal subunit joining but dispensable for the earlier steps, treatment with the nonhydrolysable GTP analog GMP-PNP arrests scanning complexes at the initiator AUG codon of the mRNA (Anthony and Merrick 1992; Pestova et al. 2000).

Translation extracts were adjusted to $2.5 \mathrm{mM}$ GMP-PNP following eIF4G cleavage and incubated with c-myc IRES mRNAs bearing or lacking a poly(A) tail. Initiation complexes were resolved through 5\%-25\% sucrose gradients to monitor $48 \mathrm{~S}$ initiation complex formation (see Materials and Methods). While $48 \mathrm{~S}$ complexes form on polyadenylated c-myc IRES mRNAs (Fig. 3A, fractions 29-36, blue line), 
A

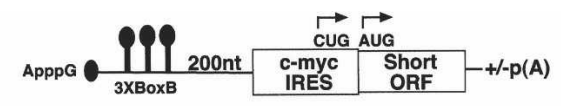

B

C

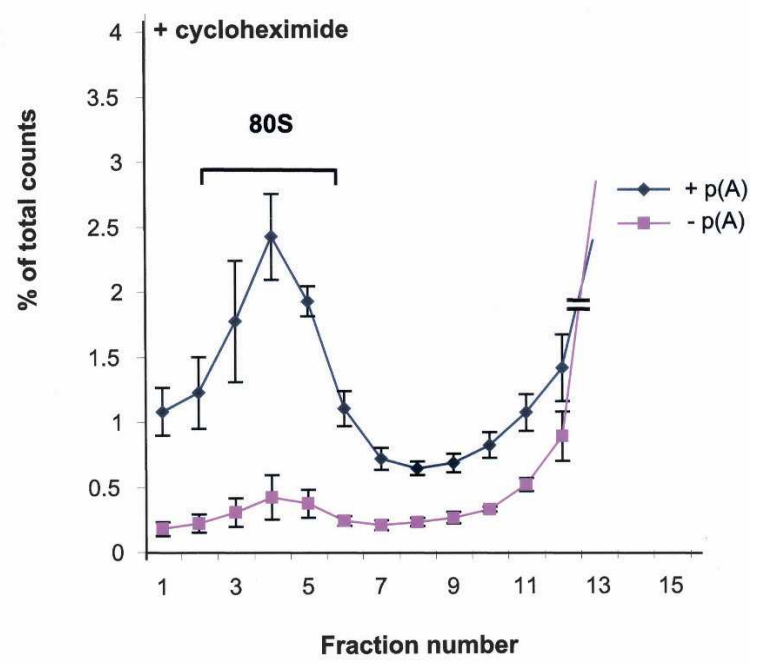

D

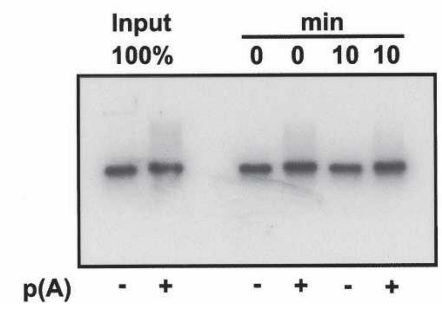

FIGURE 2. The poly(A) tail enhances 80 S initiation complex assembly on c-myc IRES mRNAs. (A) Schematic representation of the ApppG-capped c-myc IRES-containing reporter mRNAs. Three copies of the BoxB hairpin GGGCCCTGAAGAAGGGCCC (loop is in bold, double strand area is underlined) were introduced upstream of a spacer sequence and the c-myc IRES for GRNA affinity chromatography (see Fig. 4). (B) HeLa cell extracts were programmed with ApppG-capped c-myc IRES reporter mRNAs bearing or lacking an $\mathrm{A}_{(62)}$ tail. A reporter mRNA lacking the c-myc IRES upstream of the short ORF was used as a negative control. ${ }^{35}$ S-methionine and cysteine-labeled translation products were resolved by SDS-PAGE followed by autoradiography. A representative result of at least three independent experiments is shown. (C) HeLa cell extracts were preincubated with coxackievirus protease 2A. Following protease treatment, in vitro translation reactions containing ${ }^{32} \mathrm{P}$-radiolabeled ApppG-capped c-myc IRES reporter mRNAs bearing or lacking an $\mathrm{A}_{(62)}$ tail were performed in the presence of cycloheximide. The reactions were loaded onto 5\%-25\% linear sucrose density gradients, and complexes were resolved by centrifugation. Fractions were taken from the bottom of the gradient and analyzed by scintillation counting. Radioactivity is expressed as percentage of total recovered counts, plotted against the fraction number. The profile of the upper fractions from the sucrose density gradients is omitted for clarity. For each mRNA, an averaged graph of three independent experiments is shown together with an indication of the experimental variability. $(D)$ In vitro translation reactions containing ${ }^{32} \mathrm{P}$-radiolabeled ApppGcapped c-myc IRES reporter mRNAs bearing or lacking an $\mathrm{A}_{(62)}$ tail were performed in the presence of cycloheximide as in panel $C$. Total RNA was isolated before $(t 0)$ and after $(t 10)$ translation initiation and separated by gel electrophoresis followed by autoradiography.
$48 \mathrm{~S}$ complex recruitment to c-myc IRES mRNAs lacking a poly(A) tail is almost nondetectable (Fig. 3A, pink line). Taken together, these experiments show that the $\operatorname{poly}(\mathrm{A})$ tail stimulates $48 \mathrm{~S}$ ribosomal complex formation with cmyc IRES mRNAs, apparently independently of intact eIF4G.

\section{Enhancement of $48 \mathrm{~S}$ ribosomal complex formation on c-myc IRES mRNA by the poly(A) tail is independent of PABP}

Since we observed that the enhancement of IRES-mediated translation by the poly(A) tail is independent of PABP (Fig. 1 ), we wanted to examine directly whether $48 \mathrm{~S}$ initiation complex recruitment to the c-myc IRES is also PABP independent. We depleted PABP from HeLa cell extracts such that the strong poly(A) enhancement of cap-dependent translation is almost completely lost (Fig. 1C, cf. lanes 1,2 and 3,4,7,8). PABP-depleted extracts continue to show strong poly(A) enhancement of $48 \mathrm{~S}$ initiation complex formation with the c-myc IRES mRNA, albeit slightly less than mock-depleted extracts (Fig. 3B). Thus, PABP is dispensable for the poly(A)-mediated stimulation of $48 \mathrm{~S}$ complex formation with c-myc IRES mRNA, in contrast to its role in cap-dependent translation.

\section{Quantitative label-free analysis of native 485 c-myc IRES translation initiation complexes by liquid chromatography and mass spectrometry}

Viral IRES elements utilize different eIFs for translation initiation. There is currently no such information available for cellular IRESs. Therefore, we set out to analyze the composition of c-myc IRES $48 \mathrm{~S}$ translation initiation complexes assembled on the polyadenylated mRNAs. To this end, we used the three boxB RNA hairpins upstream of the IRES; as a specificity control, a c-myc IRES mRNA lacking the boxB sequences was used (Fig. 4A). The boxBcontaining c-myc RNAs were validated to initiate translation as efficiently as their counterparts lacking the boxB insertion (data not shown). Native ribosomal $48 \mathrm{~S}$ complexes were then assembled in the presence of GMP-PNP in eIF4G-cleaved and PABP-depleted HeLa cell extracts and resolved on sucrose density gradients. Nontreated extracts (i.e., no PABP depletion or eIF4G cleavage) were used as a control. 48S initiation complexes were then isolated from gradient fractions 27 to 33 by GRNA chromatography (Fig. 4A; Czaplinski et al. 2005; Duncan et al. 2006). The eluted complexes were analyzed by quantitative label-free shotgun proteomics (Fraterman et al. 2007). Importantly, this strategy allows validation of the $48 \mathrm{~S}$ ribosomal complexes by determining the enrichment of small versus large ribosomal subunit proteins. It also permits the comparative quantitative analysis of eIF association in control versus eIF4G-cleaved and PABP-depleted extracts. In particular, it 
A

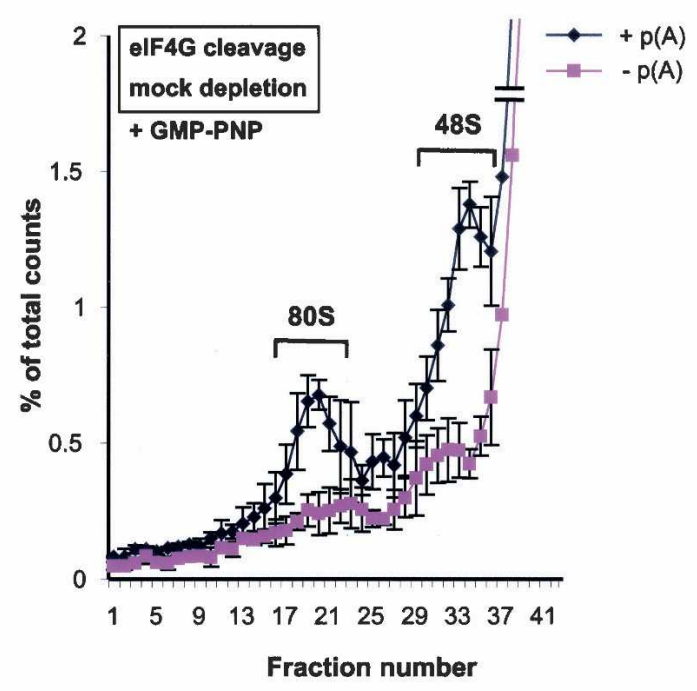

B

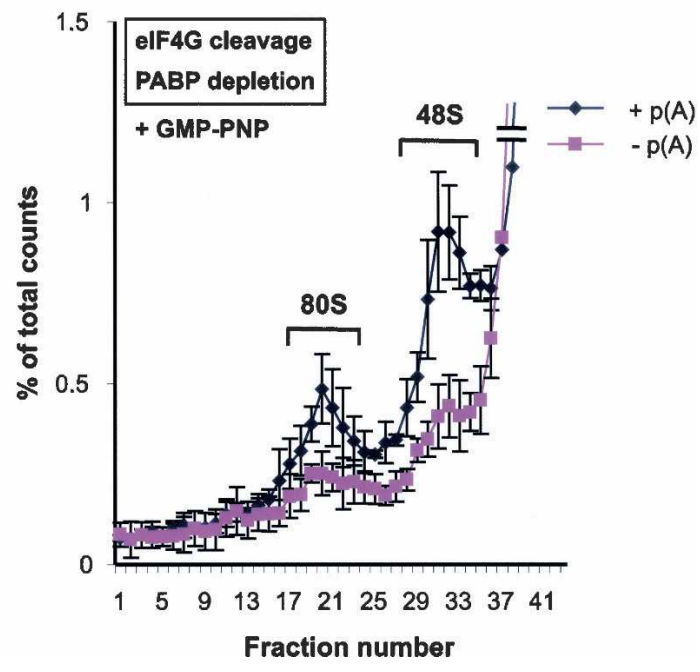

FIGURE 3. The poly(A) tail enhances $48 \mathrm{~S}$ complex formation with c-myc IRES mRNAs in a PABP-independent manner. HeLa extracts were treated as described in Figure 2C. In vitro translation reactions containing ${ }^{32} \mathrm{P}$-radiolabeled ApppG-capped c-myc IRES reporter mRNAs bearing or lacking an $\mathrm{A}_{(62)}$ tail were performed in the presence of GMP-PNP in control $(A)$ and PABP-depleted $(B)$ extracts, and analyzed as described in Figure 2C. An additional peak forms with polyadenylated c-myc mRNAs further toward the bottom of the gradient ( $A$, fractions 17-24, blue line). This minor peak may represent $80 \mathrm{~S}$ ribosomal complexes that assemble due to leakiness from incomplete replacement of endogenous GTP by GMP-PNP. This explanation is supported by an analysis of initiation complex formation on c-myc mRNAs in extracts supplemented with either cycloheximide alone or cycloheximide plus GMP-PNP (data not shown). The profile of the upper fractions from the sucrose density gradients is omitted for clarity. For each mRNA, an averaged graph of three independent experiments is shown together with an indication of the experimental variability. allows the quantitative analysis of PABP association with c-myc IRES translation initiation complexes from the two different extracts by comparison of ion volumes of BoxBcontaining samples with the "no BoxB" control samples and calculation of a "fold increase" representing the enrichment of purified proteins.

Whereas 60S ribosomal proteins are not enriched, $40 \mathrm{~S}$ ribosomal proteins are ninefold enriched on BoxBcontaining mRNAs over the "no BoxB" controls (Fig. $4 \mathrm{~B})$, showing that $48 \mathrm{~S}$ translation complexes are specifically purified. We next addressed the question of whether the effective stimulation of translation by the poly(A) tail in PABP-depleted extracts might be caused by highly efficient recruitment of residual PABP to the c-myc IRES mRNA. At the same time we explored the possibility of whether a PABP isoform that might have escaped depletion mediates the poly(A) effect. In humans, three cytoplasmic PABP isoforms (testis PABP, inducible PABP, and PABPC5) have been identified in addition to the well-studied major isoform PABPC1 (Gorgoni and Gray 2004). Although two PABP isoforms, PABPC1 and PABPC4, are identified in c-myc IRES 48S initiation complexes, recruitment of both PABPC1 and PABPC4 to polyadenylated c-myc IRES mRNA is almost abolished in eIF4G-cleaved and PABPdepleted extracts (Fig. 5A). This result implies that PABP is not a mediator of the poly(A) enhancement of cellular IRES translation. It also implicates a distinct factor(s) in the poly(A)mediated translational stimulation of cellular IRESs.

The functions of eIF4GI and eIF4GII in translation initiation were initially considered to be largly redundant (Goyer et al. 1993; Gradi et al. 1998). Our direct analyses now identify eIF4A and eIF4GI as specific components of native $48 \mathrm{~S}$ initiation complexes (Fig. 5B). These complexes appear not to contain eIF4GII (Fig. 5B), although eIF4GII is abundant in HeLa cell extracts (Hundsdoerfer et al. 2005). The abundance of eIF4GI and eIF4GII previously has been determined to be $3 \mathrm{pmol} / \mu \mathrm{L}$ HeLa cell extract for eIF4GI and $4-5 \mathrm{pmol} / \mu \mathrm{L}$ HeLa cell extract for eIF4GII (Hundsdoerfer et al. 2005). We have confirmed these quantitative data for the HeLa extracts used in this study (data not shown).

Note that eIF4GI, eIF4A, and eIF4B levels are reduced 4.9-fold, 2.9-fold, and 1.9-fold, respectively, in eIF4Gcleaved and PABP-depleted extracts compared to control extracts (Fig. 5B). This result is not a technical artifact arising from eIF4G cleavage, because the method reliably detects peptides from all regions of eIF4GI (Fig. 5C). The native IRES $48 \mathrm{~S}$ initiation complexes also contain the translation initiation factors eIF2, eIF3, and eIF5. The levels of these eIFs vary only between 1.1-fold for eIF3 and 1.4-fold for eIF5, respectively, in eIF4G-cleaved and PABP-depleted versus control extracts. Since both extracts display active IRES-mediated translation, these results directly implicate these initiation factors in the c-myc IRES-driven translation initiation mechanism. 
A
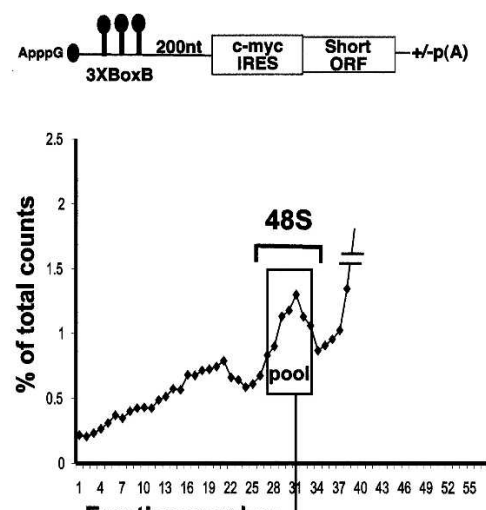
Fraction number
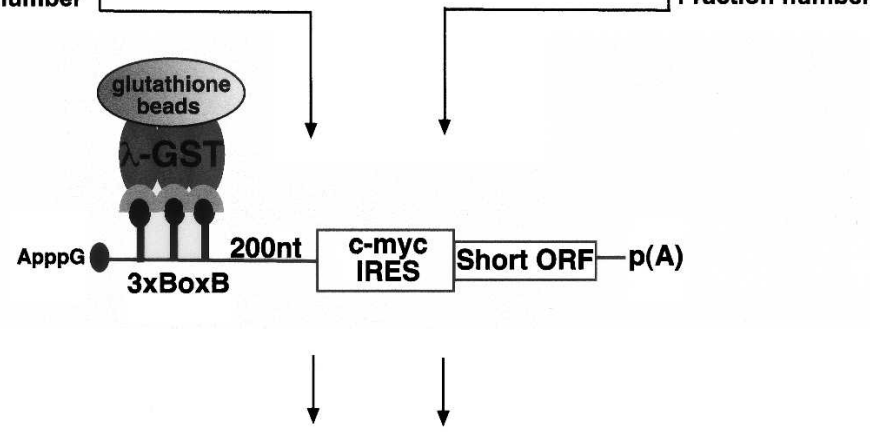

Quantitative mass spectrometry

B

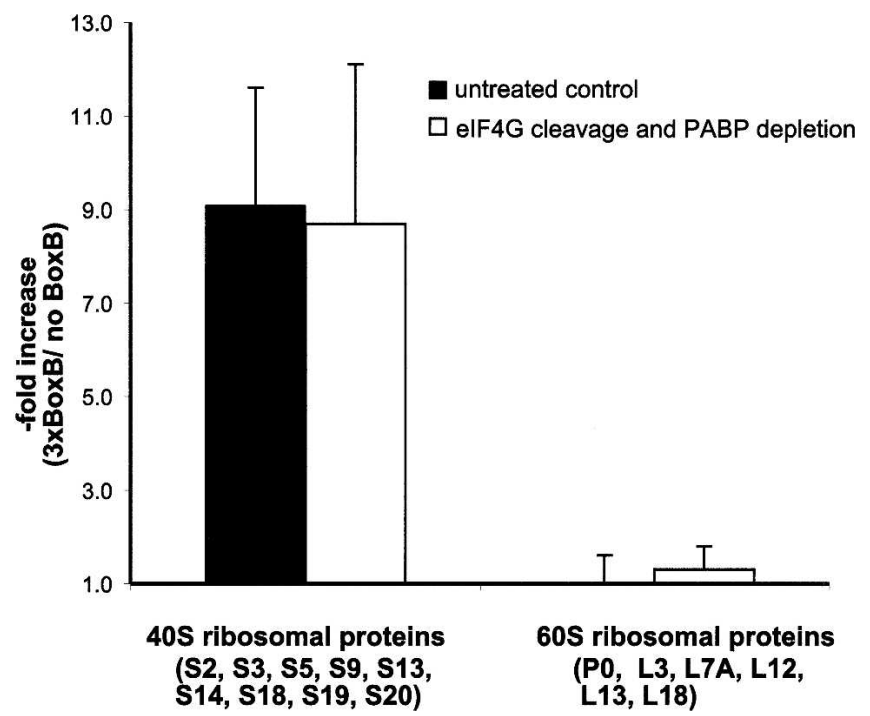

FIGURE 4. Isolation and characterization of native c-myc IRES 485 initiation complexes. $(A)$ A schematic representation of ApppG-capped c-myc IRES-containing reporter mRNAs used for GRNA affinity chromatography via the lambda phage N-protein/boxB hairpin interaction is shown above the purification scheme. Purifications were performed from control extracts and PABP-depleted extracts that were treated with coxackievirus protease 2A. Translation reactions were programmed with the indicated ${ }^{32} \mathrm{P}$-radiolabeled ApppG-capped mRNAs in the presence of GMP-PNP. The reactions were subjected to $5 \%-25 \%$ linear sucrose density gradient analysis; $48 \mathrm{~S}$ fractions were pooled prior to isolation by GRNA chromatography. The gradient profile of 3xboxB-containing c-myc mRNAs (left panel) and the negative control without the boxB elements (right panel) incubated in eIF4G-cleaved and PABP-depleted reactions is shown as an example. The eluates from the GRNA affinity chromatography were analyzed by quantitative mass spectrometry. (B) Quantitative mass spectrometry analysis of c-myc IRES translation initiation intermediates derived from control versus eIF4G-cleaved and PABP-depleted extracts. Calculated fold changes result from the comparison of 3xboxBcontaining c-myc mRNAs with the no boxB control. Error bars for $40 \mathrm{~S}$ and $60 \mathrm{~S}$ ribosomal proteins were calculated based on the fold changes of individual ribosomal proteins identified in the duplicate analyses.

\section{DISCUSSION}

The poly(A) tail is essential for c-myc IRES-driven translation in vivo (Thoma et al. 2004a). Here, we describe the underlying mechanism using a validated HeLa cell-derived in vitro translation system (Bergamini et al. 2000; Thoma et al. 2004a,b; Hundsdoerfer et al. 2005). The data reveal that the poly(A) tail enhances first round translation initiation by the c-myc IRES. Specifically, it augments $48 \mathrm{~S}$ complex recruitment and does so in a way that is distinct from its activation of cap-dependent translation initiation, independently of intact eIF4G and PABP. Analysis of native $48 \mathrm{~S}$ initiation complexes by quantitative mass spectrometry yields direct information on the composition of these particles, including the differential association of eIF4GI in comparison to eIF4GII.

The question of how the poly(A) tail enhances translation via a cellular IRES has not yet been addressed. We show that $80 \mathrm{~S}$ complex assembly is stimulated by the poly(A) tail on c-myc IRES mRNA (Fig. 2C); hence, the poly(A) tail enhances translation initiation. The quantitatively strong effect of the poly (A) tail on $80 \mathrm{~S}$ complex formation suggests that this is a major contribution to the overall translational effect (Fig. 1B). A potential additional contribution to a post-initiation step (i.e., elongation, termination) or to ribosome recycling, however, is not excluded by these data.

The marked enhancement of $48 \mathrm{~S}$ ribosome complex formation with cmyc mRNA (Fig. 3) further reveals that the poly(A) tail promotes translation initiation at an early step by increasing the stable binding of $48 \mathrm{~S}$ initiation complexes to the mRNA. Since it is unknown whether $80 \mathrm{~S}$ complexes and $48 \mathrm{~S}$ complexes are equally stable in sucrose gradient analyses, direct quantitative comparisons are difficult. Nonetheless, the poly(A) tail enhancement of both steps does not appear to differ dramatically, indicating that $60 \mathrm{~S}$ subunit joining is not a primary target.

What are the molecular requirements for this enhancer function? Intact eIF4G, 

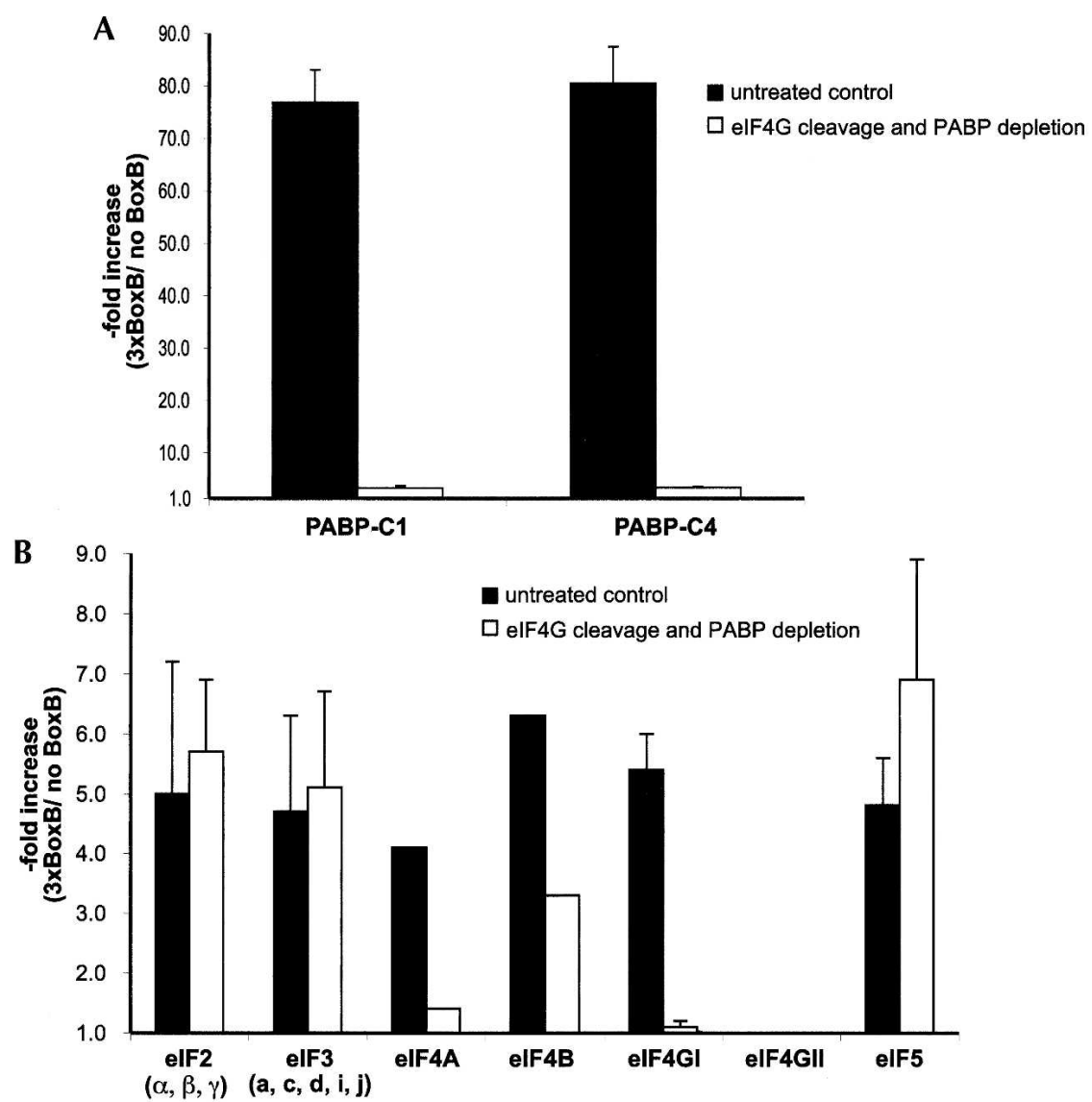

C

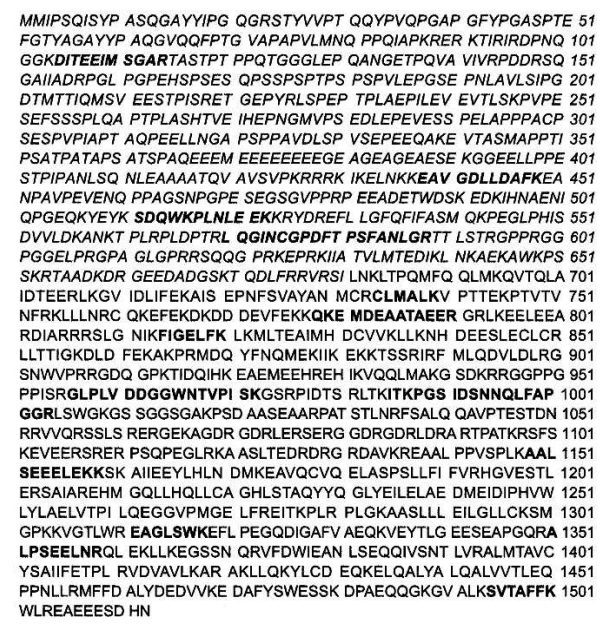

FIGURE 5. Translation factors associated with native c-myc IRES $48 \mathrm{~S}$ initiation complexes assembled in control versus eIF4G-cleaved and PABP-depleted extracts. Calculated fold changes result from the comparison of $3 \mathrm{x}$ boxB-containing mRNAs with the no boxB control. (A) Both PABPC1 and PABPC4 levels are 26-fold reduced after PABP depletion. Error bars are calculated based on the individual fold changes of each replicate. $(B)$ Error bars for eIF2 and eIF3 were calculated based on the fold changes of individual subunits identified in the repeated analysis. Error bars for the other initiation factors were calculated based on the duplicate analysis. Peptides for eIF4A and eIF4B were only identified in one of the two replicates due to undersampling of the mass spectrometer. Therefore error bars are missing. eIF4E, eIF1, eIF1A, and eIF5B were not detected. $(C)$ Primary structure of eIF4GI. The N-terminal domain is presented in italics. Peptides identified by mass spectrometry derived from control extracts are indicated in bold. which is critical for cap-dependent translation (Liebig et al. 1993), is dispensable for the stimulatory $\operatorname{poly}(\mathrm{A})$ effect on $48 \mathrm{~S}$ complex assembly on cmyc IRES mRNA (Fig. 3A). This result explains previous work showing that the enhancement of cellular IRESdriven translation by the poly(A) tail is independent of intact eIF4G (Thoma et al. 2004a).

Isolation of native translation initiation intermediates and determination of their composition could shed light on the involvement of translation initiation factors in c-myc IRES translation. To this end, we combined GRNA chromatography (Czaplinski et al. 2005; Duncan et al. 2006) with quantitative label-free shotgun mass spectrometry (Fraterman et al. 2007). Interestingly, we specifically identify eIF4GI but not eIF4GII within c-myc IRES-containing $48 \mathrm{~S}$ complexes (Fig. 5B), even though eIF4GII is as abundant as eIF4GI in our HeLa cell extracts (Hundsdoerfer et al. 2005; data not shown). Hence the two isoforms of eIF4G are not functionally redundant, at least in c-myc IRES translation. However, we cannot formally exclude the possibility that eIF4GII may have dissociated during the purification procedure.

We observe a reduced association of eIF4GI, eIF4A, and eIF4B with c-myc IRES 48S translation complexes purified from eIF4G-cleaved and PABPdepleted extracts (Fig. 5B). This is unexpected and interesting, given the importance of eIF4GI and eIF4A in c-myc IRES-driven translation (Thoma et al. 2004a; Hundsdoerfer et al. 2005). Possibly, binding of eIF4GI to the cmyc IRES-containing mRNA is weakened after cleavage, which would destabilize associated factors. It has been proposed for ${ }^{7} \mathrm{mGpppG}$-capped mRNAs that PABP increases the affinity of eIF4G for the mRNA via its interaction with the $\mathrm{N}$ terminus of eIF4G. In this case, disruption of the eIF4G/PABP interaction by eIF4G cleavage and/or PABP depletion may well affect the stability of eIF4G binding. Work on EMCV IRES-mediated initiation shows that eIF4A functions as part of a complex 
with eIF4G rather than as a singular protein (Hellen and Sarnow 2001). This may also explain the concomitant reduction of eIF4A association in our experiments. The drop of eIF4B might be a consequence of PABP depletion, as it has been shown that eIF4B interacts with PABP (Bushell et al. 2001). This interpretation points to the technical shortcoming of our approach: Weakly associated factors will likely be lost from ribosomal complexes before affinity purification and proteomic analysis because of the stringency of the sucrose density gradient step. This may account for the failure to identify eIF1 or eIF1A in the c-myc IRES-containing $48 \mathrm{~S}$ complexes (Fig. 5B). Other initiation factors (e.g., eIF 2, 3, and 5) and ribosomal proteins are not reduced (Figs. 4B, 5B), excluding differences in the purification procedure as the underlying cause for the effects on eIF4GI, eIF4A, and eIF4B. An interesting possibility that may also explain these results is that eIF4GI, eIF4A, and eIF4B may exert their function prior to the completion of $48 \mathrm{~S}$ complex assembly and then dissociate. In this scenario, their continued association with the c-myc IRES mRNAs in control extracts may reflect a stabilizing function of PABP. Taken together, the proteomic analysis of the c-myc IRES initiation intermediates further supports the notion that the poly(A) enhancement of $48 \mathrm{~S}$ complex assembly on c-myc IRES mRNA is unaffected by eIF4GI cleavage. This may explain why the c-myc IRES remains translationally active during important physiological processes when eIF4G is cleaved and cap-dependent translation is compromised during, for example, apoptosis, mitosis, and stress.

We also explored the role of PABP by the proteomic approach. Following PABP depletion from the translation extracts, both PABPC1 and PABPC4 association with polyadenylated c-myc IRES mRNA drastically is reduced (Fig. 5A), although c-myc IRES translation is even 2.5-fold increased (Fig. 1B). This result directly argues against the possibility that residual $\mathrm{PABP}$ is more effectively recruited to c-myc IRES containing mRNAs. How does the poly(A) tail enhance c-myc IRES-driven translation initiation? Analogous to the eIF4E-eIF4G-PABP bridging complex that enhances cap-dependent translation, the poly(A) tail and the c-myc IRES may be similarly bridged. It is also possible that the poly(A) tail acts by changing the mRNA secondary structure and that such a structural change contributes to enhanced (PABP-independent) c-myc IRES translation initiation. Future experiments will aim to distinguish between these possibilities.

\section{MATERIALS AND METHODS}

\section{Plasmids}

The pT3luc(pA) and pSL200Mluc(pA) plasmids have been described (Iizuka et al. 1994; Hundsdoerfer et al. 2005). To generate pBbox200MS(pA), a NcoI/SpeI fragment containing the luc ORF of pSL200Mluc(pA) was replaced with a NcoI/HpaI fragment of the plasmid pBSEF containing a synthetic short ORF (Gebauer et al. 2003) generating pSL200MS(pA). Next, three BoxB sequences derived from pBSEF-BoxB (Duncan et al. 2006) were introduced into the KpnI site of pSL200MS(pA). To obtain the "no IRES" control construct, the HindIII/NcoI fragment of Bbox200MS(pA) containg the c-myc IRES was removed. All constructs were verified by DNA sequencing. The plasmid $\mathrm{p} \lambda \mathrm{GST}$ for GRNA chromatography was kindly provided by K. Czaplinski (Czaplinski et al. 2005).

\section{In vitro transcription and translation}

In vitro transcription of mRNAs in the presence of either ${ }^{7} \mathrm{mGpppG}$ or ApppG, the preparation of HeLa cell extracts, and in vitro translation assays were described previously (Bergamini et al. 2000). We used $1.5 \mathrm{mM} \mathrm{MgOAc}$ and $60 \mathrm{mM} \mathrm{KOAc}$ in translation reactions. All reactions were performed in micrococcal nuclease-treated HeLa extracts. Micrococcal nuclease treatment and protease 2A treatment of HeLa cell extracts were described previously (Thoma et al. 2004a). The concentration of exogenous mRNA was $1 \mathrm{ng} / \mu \mathrm{L}$ for ${ }^{7} \mathrm{mGpppG}$-capped luc mRNAs, $5 \mathrm{ng} / \mu \mathrm{L}$ for ApppG-capped c-myc IRES luc mRNAs, and $15 \mathrm{ng} / \mu \mathrm{L}$ for c-myc IRES short ORF mRNAs. Translation reactions were incubated at $37^{\circ} \mathrm{C}$ for $30 \mathrm{~min}$. For labeling of proteins, $\left[{ }^{35} \mathrm{~S}\right]$ methionine $(0.75 \mathrm{mCi} / \mathrm{mL})$ and cysteine $(1.43 \mathrm{mCi} / \mathrm{mL})$ were substituted for unlabeled methionine and cysteine in the translation reaction.

\section{PABP depletion from HeLa cell extracts}

Affinity depletion of PABP using immobilized PAIP2 was performed as described (Svitkin and Sonenberg 2004; Thoma et al. 2004a).

\section{Recombinant proteins}

Coxackievirus protease $2 \mathrm{~A}$ was generously provided by the late Ernst Kuechler (Vienna, Austria). $\lambda$-GST protein was expressed in Escherichia coli and purified as described (Czaplinski et al. 2005).

\section{Western blotting and antibodies}

The following antibodies were used: monoclonal anti-eIF4GI antibody, 1:250 dilution (BD Transduction Laboratories, Pharmingen), monoclonal anti-PABP antibody 10E10, 1:3000 dilution (kindly provided by M. Görlach, Jena, Germany), and monoclonal antiactin antibody, 1:5000 dilution (Sigma). Samples were separated by SDS-PAGE and electroblotted onto PVDF membranes (Immobilon, Millipore). Protein signals were detected using enhanced chemiluminescent procedure.

\section{Sucrose density gradient analysis}

Translation initiation intermediates were assembled on radiolabeled mRNAs in 50- $\mu \mathrm{L}$ reactions. We used $1 \mathrm{mM}$ cycloheximide or $2.5 \mathrm{mM}$ GMP-PNP to stall defined initiation intermediates. Reactions were incubated for $10 \mathrm{~min}$ at $37^{\circ} \mathrm{C}$, then diluted $1: 1$ 
with sucrose gradient buffer (16 mM HEPES at pH 7.4, $60 \mathrm{mM}$ KAc, $1.5 \mathrm{mM} \mathrm{MgAc}, 1 \mathrm{mM}$ DTT), and loaded on top of linear $5 \%-25 \%$ sucrose density gradients $(4.5 \mathrm{~mL}$ for the separation of $80 \mathrm{~S}$ complexes and $10 \mathrm{~mL}$ for the separation of $48 \mathrm{~S}$ complexes). After centrifugation at 30,000 rpm for $3 \mathrm{~h}$ at $4^{\circ} \mathrm{C}$ in a SW55TI rotor $(80 \mathrm{~S})$ or $38,000 \mathrm{rpm}$ for $3 \mathrm{~h}$ at $4^{\circ} \mathrm{C}$ in a SW40 rotor (48S), respectively, fractions were collected from the bottom of the gradient and analyzed by scintillation counting.

\section{GRNA chromatography}

For native initiation complex purifications, we adapted a previously described protocol (Duncan et al. 2006). For each RNA, five separate reactions were used for sucrose gradient purifications and later pooled for mass spectrometry analysis. For each BoxBcontaining c-myc IRES mRNA, an amount corresponding to $4 \mathrm{pmol}$ was subjected to elution after purification. For each individual reaction, 45 pmol of RNAs were incubated in $500 \mu \mathrm{L}$ of in vitro translation reactions for $10 \mathrm{~min}$ at $37^{\circ} \mathrm{C}$ and subsequently loaded onto a $10 \mathrm{~mL}$ linear sucrose density gradient. After centrifugation at $38,000 \mathrm{rpm}$ for $3 \mathrm{~h}$, fractions were collected from the bottom. $48 \mathrm{~S}$ fractions (Fig. 4A) of each gradient were pooled. One milliliter of pooled fractions was supplemented with heparin, NP-40, and glycerol to a final concentration of $12 \mu \mathrm{g} / \mathrm{mL}, 0.05 \%, 8.7 \%$, respec- tively, and added to $60 \mu \mathrm{L}$ of glutathione-Sepharose (Amersham) prebound to $35 \mu \mathrm{g} \lambda$-GST protein. Samples were incubated for $3 \mathrm{~h}$ with end-over-end rotation, then washed three times with $400 \mu \mathrm{L}$ of ice-cold binding buffer (Duncan et al. 2006). Counts associated with beads were measured by scintillation counting. RNA-associated proteins were eluted by adding $10 \mu \mathrm{L}$ ice-cold binding buffer and $0.7 \mu \mathrm{L}$ bovine pancreatic RNase A $(100 \mu \mathrm{g} / \mathrm{mL}$ stock, protease-free, Calbiochem) to the beads and incubating at $30^{\circ} \mathrm{C}$ for $30 \mathrm{~min}$ with shaking. Eluates were precipitated with methanol and chloroform and processed by label-free quantitative mass spectrometry.

\section{Label-free quantitative mass spectrometry}

Peptides were trypsin-digested in solution and separated on a nano-flow 1D-plus Eksigent HPLC system coupled with a QStar Pulsar $i$ quadrupole Time-of-Flight MS (Applied Biosystemsy) as described (Fraterman et al. 2007). Peptides were identified by searching the peak-list against the IPI human (3.19_20060712, 60,937 sequence entries) database using the MASCOT v2.103 (Matrix Science) algorithm and standard search parameters. All peptides were identified with a MASCOT score above 18. Peptide sequences were checked for isoform specificity as previously described (Fraterman et al. 2007). For each protein the number of fragment spectra matched by MASCOT is shown in Table 1.

TABLE 1. Protein identifications of GRNA chromatography eluates

\begin{tabular}{|c|c|c|c|}
\hline Protein & Accession no. & Molecular weight & Queries matched \\
\hline Polyadenylate-binding protein 1 & IPI00008524 & 70,854 & 49 \\
\hline Polyadenylate-binding protein 4 & IPI00012726 & 71,080 & 37 \\
\hline Eukaryotic translation initiation factor 3 a & IPI00029012 & 168,677 & 22 \\
\hline 40S ribosomal protein S18 & IPI00013296 & 17,708 & 19 \\
\hline Eukaryotic translation initiation factor 4 gamma 1 & IPI00220365 & 155,450 & 16 \\
\hline $40 \mathrm{~S}$ ribosomal protein $\mathrm{S} 3$ & IPI00011253 & 26,842 & 13 \\
\hline Eukaryotic translation initiation factor $3 \mathrm{c}$ & IPI00016910 & 105,962 & 13 \\
\hline $40 S$ ribosomal protein S2 & IPI00013485 & 31,590 & 12 \\
\hline $40 S$ ribosomal protein 59 & IPI00221088 & 22,504 & 12 \\
\hline Eukaryotic translation initiation factor $2 \beta$ & IPI00021728 & 38,707 & 11 \\
\hline 40S ribosomal protein S19 & IPI00215780 & 15919 & 10 \\
\hline Eukaryotic translation initiation factor $2 \gamma$ & IPI00297982 & 51,516 & 10 \\
\hline $40 \mathrm{~S}$ ribosomal protein S6 & IPI00021840 & 28,834 & 9 \\
\hline 40 S ribosomal protein S20 & IPI00012493 & 13,478 & 7 \\
\hline Eukaryotic translation initiation factor $2 \alpha$ & IPI00398135 & 16,726 & 7 \\
\hline Eukaryotic translation initiation factor $3 \mathrm{~d}$ & IPI00006181 & 64,560 & 6 \\
\hline $40 \mathrm{~S}$ ribosomal protein $\mathrm{S} 13$ & IPI00221089 & 17,081 & 5 \\
\hline $40 \mathrm{~S}$ ribosomal protein S5 & IPI00008433 & 22,902 & 5 \\
\hline Eukaryotic translation initiation factor 5 & IPI00022648 & 49,648 & 5 \\
\hline $40 \mathrm{~S}$ ribosomal protein $\mathrm{S} 14$ & IPI00026271 & 16,303 & 4 \\
\hline Eukaryotic translation initiation factor $3 \mathrm{i}$ & IPI00012795 & 36,818 & 4 \\
\hline $60 S$ ribosomal protein $\mathrm{P} 0$ & IPI00008530 & 34,423 & 3 \\
\hline $60 S$ ribosomal protein L12 & IPI00024933 & 17,979 & 3 \\
\hline 60 S ribosomal protein L13 & IPI00465361 & 24,173 & 3 \\
\hline Eukaryotic initiation factor $4 \mathrm{~A}$ & IPI00025491 & 46,353 & 3 \\
\hline $60 S$ ribosomal protein L3 & IPI00055021 & 46,234 & 3 \\
\hline Eukaryotic translation initiation factor $3 \mathrm{j}$ & IPI00012795 & 36,878 & 3 \\
\hline $60 S$ ribosomal protein L18 & IPI00026202 & 21,034 & 2 \\
\hline $60 S$ ribosomal protein $\mathrm{L} 7 \mathrm{a}$ & IPI00299573 & 30,017 & 2 \\
\hline Eukaryotic translation initiation factor $4 \mathrm{~B}$ & IPI00012079 & 69,183 & 2 \\
\hline
\end{tabular}

For each protein identified in the study, protein name, accession number, molecular weight in Daltons, and the number of queries matched by MASCOT search engine are presented. 
Quantitative analysis was performed using MSQuant in a no label setting (Schulze and Mann 2004). The quantitation results (peptide ion volumes in Thompson-seconds) for differentially expressed peptides were visually inspected for anomalies. To retrieve quantitative data for peptides that were only identified in a single experiment, the resulting MASCOT search result file was cross-correlated with generic mass spectrometric data from other samples (Pasa-Tolic et al. 2004). Protein ion volumes were based on the average ion volumes of two different peptide ion volumes. The presented "fold increase" is the ratio of the ion volumes of the BoxB-containing samples to the no BoxB control samples.

For eIF2, eIF3, 40S, and 60S ribosomal proteins, an average of the individual subunit protein fold increases was calculated and presented. The presented quantitative result represents the average of two independent experimental replicates. Peptides for eIF4A and eIF4B were only identified in one of the two replicates due to undersampling of the mass spectrometer and therefore only a single replicate was used to calculate the quantitative information. Standard deviations were calculated using Microsoft Excel (Microsoft) and presented as error bars (Fraterman et al. 2007).

\section{ACKNOWLEDGMENTS}

We thank past and present members of the Hentze laboratory for helpful discussions and K. Duncan and R. Thermann for comments on the manuscript. We thank our colleagues K. Czaplinski, M. Görlach, and A.C. Prats for their generous gift of valuable reagents. C.T. was funded by postdoctoral fellowships from the Fritz-Thyssen-Stiftung and the Deutsche Forschungsgemeinschaft; M.W.H gratefully acknowledges funding from the Deutsche Forschungsgemeinschaft.

Received February 27, 2008; accepted April 21, 2008.

\section{REFERENCES}

Anthony, D.D. and Merrick, W.C. 1992. Analysis of 40S and 80S complexes with mRNA as measured by sucrose density gradients and primer extension inhibition. J. Biol. Chem. 267: 1554-1562.

Bergamini, G., Preiss, T., and Hentze, M.W. 2000. Picornavirus IRESes and the poly(A) tail jointly promote cap-independent translation in a mammalian cell-free system. RNA 6: 1781-1790.

Bradrick, S.S., Dobrikova, E.Y., Kaiser, C., Shveygert, M., and Gromeier, M. 2007. Poly(A)-binding protein is differentially required for translation mediated by viral internal ribosome entry sites. RNA 13: 1582-1593.

Braunstein, S., Karpisheva, K., Pola, C., Goldberg, J., Hochman, T., Yee, H., Cangiarella, J., Arju, R., Formenti, S.C., and Schneider, R.J. 2007. A hypoxia-controlled cap-dependent to cap-independent translation switch in breast cancer. Mol. Cell 28: 501-512.

Bushell, M., McKendrick, L., Janicke, R.U., Clemens, M.J., and Morley, S.J. 1999. Caspase-3 is necessary and sufficient for cleavage of protein synthesis eukaryotic initiation factor $4 \mathrm{G}$ during apoptosis. FEBS Lett. 451: 332-336.

Bushell, M., Wood, W., Carpenter, G., Pain, V.M., Morley, S.J., and Clemens, M.J. 2001. Disruption of the interaction of mammalian protein synthesis eukaryotic initiation factor $4 \mathrm{~B}$ with the poly $(\mathrm{A})$ binding protein by caspase- and viral protease-mediated cleavages. J. Biol. Chem. 276: 23922-23928.

Chappell, S.A., LeQuesne, J.P., Paulin, F.E., deSchoolmeester, M.L., Stoneley, M., Soutar, R.L., Ralston, S.H., Helfrich, M.H., and Willis, A.E. 2000. A mutation in the c-myc-IRES leads to enhanced internal ribosome entry in multiple myeloma: A novel mechanism of oncogene de-regulation. Oncogene 19: 4437-4440.

Cobbold, L.C., Spriggs, K.A., Haines, S.J., Dobbyn, H.C., Hayes, C., de Moor, C.H., Lilley, K.S., Bushell, M., and Willis, A.E. 2008. Identification of IRES-trans-acting factors for the Myc family of IRESs. Mol. Cell. Biol. 28: 40-49.

Cornelis, S., Bruynooghe, Y., Denecker, G., Van Huffel, S., Tinton, S., and Beyaert, R. 2000. Identification and characterization of a novel cell cycle-regulated internal ribosome entry site. Mol. Cell 5: 597605.

Czaplinski, K., Köcher, T., Schelder, M., Segref, A., Wilm, M., and Mattaj, I.W. 2005. Identification of $40 \mathrm{LoVe}$, a Xenopus hnRNP D family protein involved in localizing a TGF- $\beta$-related mRNA during oogenesis. Dev. Cell 8: 505-515.

Dorner, A.J., Semler, B.L., Jackson, R.J., Hanecak, R., Duprey, E., and Wimmer, E. 1984. In vitro translation of poliovirus RNA: Utilization of internal initiation sites in reticulocyte lysate. J. Virol. 50: $507-514$.

Duncan, K., Grskovic, M., Strein, C., Beckmann, K., Niggeweg, R., Abaza, I., Gebauer, F., Wilm, M., and Hentze, M.W. 2006. Sexlethal imparts a sex-specific function to UNR by recruiting it to the msl-2 mRNA 3' UTR: Translational repression for dosage compensation. Genes \& Dev. 20: 368-379.

Eisenman, R.N. 2001. Deconstructing myc. Genes \& Dev. 15: 20232030.

Evan, G. and Littlewood, T. 1998. A matter of life and cell death. Science 281: 1317-1322.

Fernandez, J., Yaman, I., Mishra, R., Merrick, W.C., Snider, M.D., Lamers, W.H., and Hatzoglou, M. 2001. Internal ribosome entry site-mediated translation of a mammalian mRNA is regulated by amino acid availability. J. Biol. Chem. 276: 12285-12291.

Fraterman, S., Zeiger, U., Khurana, T.S., Wilm, M., and Rubinstein, N.A. 2007. Quantitative proteomics profiling of sarcomere associated proteins in limb and extraocular muscle allotypes. Mol. Cell. Proteomics 6: 728-737.

Gallie, D.R. 1991. The cap and poly(A) tail function synergistically to regulate mRNA translational efficiency. Genes \& Dev. 5: 2108-2116.

Gebauer, F., Grskovic, M., and Hentze, M.W. 2003. Drosophila sexlethal inhibits the stable association of the $40 \mathrm{~S}$ ribosomal subunit with msl-2 mRNA. Mol. Cell 11: 1397-1404.

Gilbert, W.V., Zhou, K., Butler, T.K., and Doudna, J.A. 2007. Capindependent translation is required for starvation-induced differentiation in yeast. Science 317: 1224-1227.

Gingras, A.C., Raught, B., and Sonenberg, N. 1999. eIF4 initiation factors: Effectors of mRNA recruitment to ribosomes and regulators of translation. Annu. Rev. Biochem. 68: 913-963.

Gorgoni, B. and Gray, N.K. 2004. The roles of cytoplasmic poly(A)binding proteins in regulating gene expression: A developmental perspective. Brief. Funct. Genomic. Proteomic. 3: 125-141.

Goyer, C., Altmann, M., Lee, H.S., Blanc, A., Deshmukh, M., Woolford Jr., J.L., Trachsel, H., and Sonenberg, N. 1993. TIF4631 and TIF4632: Two yeast genes encoding the highmolecular-weight subunits of the cap-binding protein complex (eukaryotic initiation factor $4 \mathrm{~F}$ ) contain an RNA recognition motif-like sequence and carry out an essential function. Mol. Cell. Biol. 13: 4860-4874.

Gradi, A., Imataka, H., Svitkin, Y.V., Rom, E., Raught, B., Morino, S., and Sonenberg, N. 1998. A novel functional human eukaryotic translation initiation factor 4G. Mol. Cell. Biol. 18: 334-342.

Hellen, C.U. and Sarnow, P. 2001. Internal ribosome entry sites in eukaryotic mRNA molecules. Genes \& Dev. 15: 1593-1612.

Hundsdoerfer, P., Thoma, C., and Hentze, M.W. 2005. Eukaryotic translation initiation factor 4GI and p97 promote cellular internal ribosome entry sequence-driven translation. Proc. Natl. Acad. Sci. 102: 13421-13426.

Iizuka, N., Najita, L., Franzusoff, A., and Sarnow, P. 1994. Capdependent and cap-independent translation by internal initiation of mRNAs in cell extracts prepared from Saccharomyces cerevisiae. Mol. Cell. Biol. 14: 7322-7330. 
Jackson, R.J. and Kaminski, A. 1995. Internal initiation of translation in eukaryotes: The picornavirus paradigm and beyond. RNA 1: 985-1000.

Jang, S.K., Kräusslich, H.G., Nicklin, M.J., Duke, G.M., Palmenberg, A.C., and Wimmer, E. 1988. A segment of the 5' nontranslated region of encephalomyocarditis virus RNA directs internal entry of ribosomes during in vitro translation. J. Virol. 62: 2636-2643.

Johannes, G. and Sarnow, P. 1998. Cap-independent polysomal association of natural mRNAs encoding c-myc, BiP, and eIF4G conferred by internal ribosome entry sites. RNA 4: 15001513.

Kahvejian, A., Svitkin, Y.V., Sukarieh, R., Mboutchou, M.N., and Sonenberg, N. 2005. Mammalian poly(A)-binding protein is a eukaryotic translation initiation factor, which acts via multiple mechanisms. Genes \& Dev. 19: 104-113.

Khaleghpour, K., Svitkin, Y.V., Craig, A.W., DeMaria, C.T., Deo, R.C., Burley, S.K., and Sonenberg, N. 2001. Translational repression by a novel partner of human poly(A) binding protein, Paip2. Mol. Cell 7: 205-216.

Kim, J.H., Paek, K.Y., Choi, K., Kim, T.D., Hahm, B., Kim, K.T., and Jang, S.K. 2003. Heterogeneous nuclear ribonucleoprotein C modulates translation of c-myc mRNA in a cell cycle phasedependent manner. Mol. Cell. Biol. 23: 708-720.

Kolupaeva, V.G., de Breyne, S., Pestova, T.V., and Hellen, C.U. 2007. In vitro reconstitution and biochemical characterization of translation initiation by internal ribosomal entry. Methods Enzymol. 430: 409-439.

Liebig, H.D., Ziegler, E., Yan, R., Hartmuth, K., Klump, H., Kowalski, H., Blaas, D., Sommergruber, W., Frasel, L., Lamphear, B., et al. 1993. Purification of two picornaviral 2A proteinases: Interaction with eIF- $4 \gamma$ and influence on in vitro translation. Biochemistry 32: 7581-7588.

Marissen, W.E. and Lloyd, R.E. 1998. Eukaryotic translation initiation factor $4 \mathrm{G}$ is targeted for proteolytic cleavage by caspase 3 during inhibition of translation in apoptotic cells. Mol. Cell. Biol. 18: 7565-7574.

Michel, Y.M., Borman, A.M., Paulous, S., and Kean, K.M. 2001. Eukaryotic initiation factor 4G-poly(A) binding protein interaction is required for poly(A) tail-mediated stimulation of picornavirus internal ribosome entry segment-driven translation but not for X-mediated stimulation of hepatitis C virus translation. Mol. Cell. Biol. 21: 4097-4109.

Munroe, D. and Jacobson, A. 1990. mRNA poly(A) tail, a 3 ' enhancer of translational initiation. Mol. Cell. Biol. 10: 3441-3455.

Pasa-Tolić, L., Masselon, C., Barry, R.C., Shen, Y., and Smith, R.D. 2004. Proteomic analyses using an accurate mass and time tag strategy. Biotechniques 37: 621-624, 626-633, 636.

Pelletier, J. and Sonenberg, N. 1988. Internal initiation of translation of eukaryotic mRNA directed by a sequence derived from poliovirus RNA. Nature 334: 320-325.

Pestova, T.V., Lomakin, I.B., Lee, J.H., Choi, S.K., Dever, T.E., and Hellen, C.U. 2000. The joining of ribosomal subunits in eukaryotes requires eIF5B. Nature 403: 332-335.

Pilipenko, E.V., Pestova, T.V., Kolupaeva, V.G., Khitrina, E.V., Poperechnaya, A.N., Agol, V.I., and Hellen, C.U. 2000. A cell cycle-dependent protein serves as a template-specific translation initiation factor. Genes \& Dev. 14: 2028-2045.

Preiss, T. and Hentze, M.W. 1998. Dual function of the messenger RNA cap structure in poly(A)-tail-promoted translation in yeast. Nature 392: 516-520.

Pyronnet, S., Pradayrol, L., and Sonenberg, N. 2000. A cell cycledependent internal ribosome entry site. Mol. Cell 5: 607-616.

Sachs, A.B. and Davis, R.W. 1989. The poly(A) binding protein is required for poly(A) shortening and $60 \mathrm{~S}$ ribosomal subunitdependent translation initiation. Cell 58: 857-867.

Sarnow, P. 1989. Translation of glucose-regulated protein 78/immunoglobulin heavy-chain binding protein mRNA is increased in poliovirus-infected cells at a time when cap-dependent translation of cellular mRNAs is inhibited. Proc. Natl. Acad. Sci. 86: 5795-5799.

Schulze, W.X. and Mann, M. 2004. A novel proteomic screen for peptide-protein interactions. J. Biol. Chem. 279: 10756-10764.

Searfoss, A., Dever, T.E., and Wickner, R. 2001. Linking the 3' poly(A) tail to the subunit joining step of translation initiation: Relations of Pab1p, eukaryotic translation initiation factor 5b (Fun12p), and Ski2p-Slh1p. Mol. Cell. Biol. 21: 4900-4908.

Stoneley, M. and Willis, A.E. 2004. Cellular internal ribosome entry segments: Structures, trans-acting factors and regulation of gene expression. Oncogene 23: 3200-3207.

Stoneley, M., Chappell, S.A., Jopling, C.L., Dickens, M., MacFarlane, M., and Willis, A.E. 2000. c-Myc protein synthesis is initiated from the internal ribosome entry segment during apoptosis. Mol. Cell. Biol. 20: 1162-1169.

Subkhankulova, T., Mitchell, A., and Willis, A.E. 2001. Internal ribosome entry segment-mediated initiation of c-Myc protein synthesis following genotoxic stress. Biochem. J. 359: 183-192.

Svitkin, Y.V. and Sonenberg, N. 2004. An efficient system for cap- and poly(A)-dependent translation in vitro. Methods Mol. Biol. 257: $155-170$.

Svitkin, Y.V., Imataka, H., Khaleghpour, K., Kahvejian, A., Liebig, H.D., and Sonenberg, N. 2001. Poly(A)-binding protein interaction with elF4G stimulates picornavirus IRES-dependent translation. RNA 7: 1743-1752.

Tarun Jr., S.Z. and Sachs, A.B. 1995. A common function for mRNA 5' and 3' ends in translation initiation in yeast. Genes \& Dev. 9: 2997-3007.

Thoma, C., Bergamini, G., Galy, B., Hundsdoerfer, P., and Hentze, M.W. 2004a. Enhancement of IRES-mediated translation of the c-myc and BiP mRNAs by the poly(A) tail is independent of intact eIF4G and PABP. Mol. Cell 15: 925-935.

Thoma, C., Ostareck-Lederer, A., and Hentze, M.W. 2004b. A poly(A) tail-responsive in vitro system for cap- or IRES-driven translation from HeLa cells. Methods Mol. Biol. 257: 171-180.

Uchida, N., Hoshino, S., Imataka, H., Sonenberg, N., and Katada, T. 2002. A novel role of the mammalian GSPT/eRF3 associating with poly(A)-binding protein in $\mathrm{Cap} / \mathrm{Poly}(\mathrm{A})$-dependent translation. J. Biol. Chem. 277: 50286-50292.

Wells, S.E., Hillner, P.E., Vale, R.D., and Sachs, A.B. 1998. Circularization of mRNA by eukaryotic translation initiation factors. Mol. Cell 2: 135-140.

Wilson, J.E., Pestova, T.V., Hellen, C.U., and Sarnow, P. 2000. Initiation of protein synthesis from the A site of the ribosome. Cell 102: 511-520. 

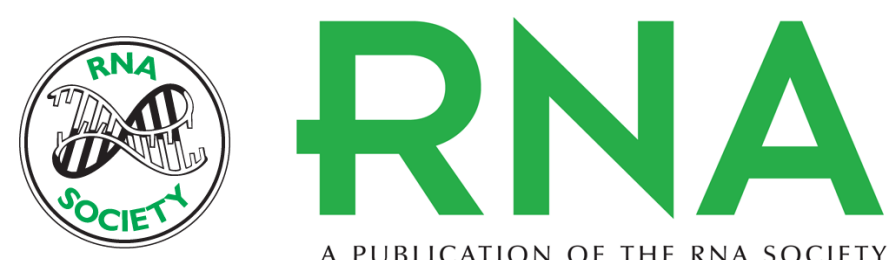

A PUBLICATION OF THE RNA SOCIETY

\section{Translation initiation by the c-myc mRNA internal ribosome entry sequence and the $\operatorname{poly}(\mathrm{A})$ tail}

Christian Thoma, Sven Fraterman, Marc Gentzel, et al.

RNA 2008 14: 1579-1589

References This article cites 58 articles, 32 of which can be accessed free at: http://rnajournal.cshlp.org/content/14/8/1579.full.html\#ref-list-1

\section{License}

Email Alerting Receive free email alerts when new articles cite this article - sign up in the box at the Service top right corner of the article or click here.

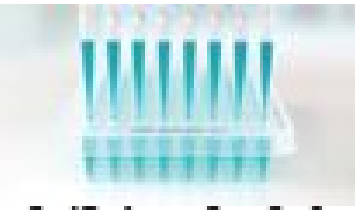

Providing Precise Solutions for your research.

To subscribe to RNA go to:

http://rnajournal.cshlp.org/subscriptions 